\title{
Threshold Oriented Preemptive Link Repair Decision based Approach for AODV in MANET
}

\author{
Shikha Patle \\ Research Scholar, Dept. of CSE \\ Oriental University \\ Indore (M.P), India
}

\author{
Purushottam Kumar \\ Assistant Prof., Dept. of CSE \\ Oriental University \\ Indore (M.P), India
}

\begin{abstract}
The routing data for associate infrastructure less network of Mobile Ad-hoc network is taken into account as an advanced task. Various protocols deal with this routing update like AODV, DSR, etc. All the way through this route construction generally the route failure happens \& route repairing mechanism must be initiated whenever it's needed. Currently this unsuccessful route must get replaced before the loss of information. In this method considering AODV there are 2 approaches that touch upon this link breakage. Link breaks between the routes may be handled either by reformation of latest shortest route from the supply to destination is made using intermediate. One among the key challenges during this context is that that one among the 2 is best and once to use that of the 2 route repair ways.In the existing approach to deciding in which condition to invoke Local Repair or Source Repair standard AODV uses an unchanging hop count based threshold as a basis in case of a link breakdown. With this type of repairing the mechanism problem arises is that in that type of network we cannot decide what algorithms to be applied at which point. For that reason to take decision same condition has to be identified. Herein also we should ensure that the decision of route repair mechanism must be pre-emptive so that loss of data can be avoided earlier than occurrence. This work proposes a novel Threshold oriented Preemptive Route Repair Algorithm for Multi-Hop Local repair in ADOV for MANET. In this proposed work focus is on early identification of link failure before its occurrence and network must know when to start route discovery if the link break occurs in the network.
\end{abstract}

\section{General Term}

MANET, AODV,DSR,Algorithm

\section{Keywords}

Local Repair, TAB (Threshold Alarm Based), PRRA (Preemptive Route Repair Algorithm), RREQ, RREP

\section{INTRODUCTION}

Mobile ad-hoc network is a wireless network, which supports the short range communication between the mobile devices. It is a self manageable network with a fixed band that conveys the packets of data and control over the remote locations with the help of intermediate devices present in their range. It is mainly helpful in the situations here the infrastructural installation is costly or cannot be provided due to regional complexities. Thus, in such situations MANET provides the required connectivity supports with its implementations.

Autonomous each type of network, whether it is wired or wireless can require routing protocols which is responsible for handling various conditions of communications in the network. Routing here faces the dynamic optimization problems because of its very small search space and changes with time due to nodes mobility. Here each node will act as a router itself installed with light weighted protocols and other applications. It demands the decentralized routing mediums which are capable of providing the support in dynamically changing topologies. Mainly the routing in MANET is classified into two categories Proactive and Reactive [1]. Here the proactive routing is a kind of table driven routing, which maintains the routing information between the various nodes in the network and supports the periodic broadcast for updating the routing table. While in reactive routing protocols the information is exchanged as per the demands of the network.

Here the routing mechanism always suffers from the changing topologies of the network due to uncontrolled nodes mobility, variable wireless bandwidth and dependent battery power. Thus a key challenge in such network is to design a mechanism which is capable of serving all these needs of dynamic topologies. Mainly the route oriented problems always affect the communication because due to any of the above factors the connectivity of the nodes is dropped and hence the communication is barded. Thus, some of the mechanism needs to be developed for handling of these events. Normally these problems are taken over by the route discovery and handling.

This work focuses its area towards improving the efficiency and reliability of on-demand routing which is a reactive category. Thus the protocol used for proving the study and other factors is based on AODV routing. It has source initiated route discovery process which sends a route request (RREQ) for identifying the nodes in communication range and updating their routing table. After sending the route request the nodes waits for a route reply (RREP) for shortest path and other hop wise detections. These replies are sometimes affected by latency and other failed links and misdirect the communications. Thus the route optimization and its effectiveness depend on the links connectivity and node mobility.

Many protocols are present, those execute on this dynamic routing behavior of the MANET. Throughout this route discovery the routing table is created and updated by neighbor $\&$ intermediate node both for starting \& end node. Due to some factors connection link to be failed or down. By Source repair or local repair this link failure can be handled depends on the link break point. The triggering condition of starting those algorithms is not known by node because it is a decision making problem. The contribution of this work is to provide Local Repair and Backup Route of Next-hop based improvements for the existing AODV routing protocol. The core algorithm of the AODV protocol is discussed in detail which is followed by the changes suggested by our 
improvements. Predictions are made for each of these improvements and compared to the simulation results run in network simulator ns-2.35. It is shown that both Local Repair and Backup Route of Next-hop based improvements result in improving the quality of the AODV protocol by increasing the number of successfully delivered packets and the packet delivery ratio.

\section{BACK GROUND}

With MANET serves various remote communication applications and processes which requires intermediate nodes for data transmission from source to destinations. The protocol which supports these operat6ons needs to be of robust types which can handle any uncertain situation communing during the transmissions. Mainly these occur due to dynamically changing topologies causes by node movements. It could within the region might be out of the region. The protocol here works towards proving the continuity of the communication by shifting the route from one intermediate node to another if the nodes move out of the regions and is a continuous process. This work uses AODV routing protocol for this reactive routing. As the works focus towards maintaining the continuity of the communication, AODV uses link repair phenomenon. If the link repair is not achieving the desired performance, then it could even degrade the networks working more than any previous conditions.

As Mobile Ad hoc Networks (MANETs) continues to experience increasing popularity, several different protocols have been proposed to efficiently transmit data among the participating nodes. These protocols have to be robust and flexible to respond to the dynamic topology and decentralized nature of MANETS. In the AODV Routing Protocol is one of the most commonly used reactive protocols for routing information in MANETS. Even though AODV performs well, it suffers from several shortcomings. This thesis aims to modify and upgrade the performance of AODV. Two different schemes are proposed which improve upon different aspects of the standard AODV routing protocol. In the first scheme, the AODV protocol is improved by adding the Local Repair feature. In this protocol, intermediate nodes in existing paths try to find new paths to the destination in the event of a link breakage. In the second scheme, Next-hop Backup Route is introduced. According to this scheme, once a path is established, every upstream node on an active route creates a backup path to its next-hop node. Thus, when link breakage happens, the upstream node can depend upon its backup node to forward the packet to the node that was previously its next hop. Both these schemes increase the number of data packets successfully transmitted to the end(destination) node.

\section{Ad hoc On-Demand Distance Vector Routing:}

Ad hoc On Demand Distance Vector Routing protocol is a single path, Loop-free protocol [2]. AODV is a combination of DSDV and DSR with the concept of sequence numbers from DSDV and on demand route discovery mechanism of DSR. AODV does not take source routing; it takes a hop-byhop routing approach. In the following section we discussed some important features of the AODV protocol required for the development of extended AODV which is AOMDV.

\section{Route detection and Route Maintenance:}

In an on-interest conventions, course revelation technique are utilized by hubs, to get courses on an 'as required' premise. In AODV, course revelation acts as takes after. At whatever point an active source needs a course to an objective, it starts a course revelation by flooding a course, ask for (RREQ) for the terminus in the system and after that holds up for a course answer (RREP). At the point when a transit hub gets the first duplicate of a RREQ bundle, it sets up a conversational way to the source utilizing the past jump of the RREQ as the following bounce on the opposite way. Moreover, if there is a legitimate course accessible for the objective, it unicasts a RREP again to the source by means of the converse way; else, it re-telecasts the RREQ parcel. Copy duplicates of the RREQ are quickly disposed of upon gathering at each hub. The end of getting the first duplicate of a RREQ parcel structure an opposite way in the same path as the middle hubs; it likewise unicasts a RREP over to the source along the converse way. As the RREP continues towards the source, it makes a forward way to the objective at each one jump. Course support is carried out by method for the course lapse (RERR) parcels. At the point when a middle hub identifies a connection disappointment (by means of a connection layer input, e.g.), it creates a RERR bundle. The RERR engenders towards all activity sources having a course through the fizzled connection, and deletes all broken courses on the way. A source after getting the RERR starts another course revelation on the off chance that regardless it needs the course. Separated from this course upkeep system, AODV additionally has a clock based component to cleanse stale courses.

\section{Analysis of Local Link Repairing Protocol:}

By the help of above discussed control packets an on demand route request is initiated by AODV be sending a Route request (RREQ) packets and get the available path by Route reply (RREP) packets. With the help of sequence number given to the node cleanness of the route is maintained. By sending hello message in the network Error in route is detected; in case of error detection the node which detects the error will generate a RERR message in the network. In existing AODV there is no provision for fast and localized route recovery method and to find alternate path in between the transmission link as shown in figure 1. Many researchers contributed in the field of route maintenance if there is a link breakage during the transmission of data in the network [5].

In this work we are going to propose an extended AODV routing protocol (AOMDV) for mobile ad hoc networks. In this approach we are going to propose multipath extensions to a existing well suited single path and Loop-free routing protocol which is ad hoc on-demand distance vector (AODV) routing protocol [6]. In the proposed approach modified AODV guarantees loop freedom and explicit alternate paths.

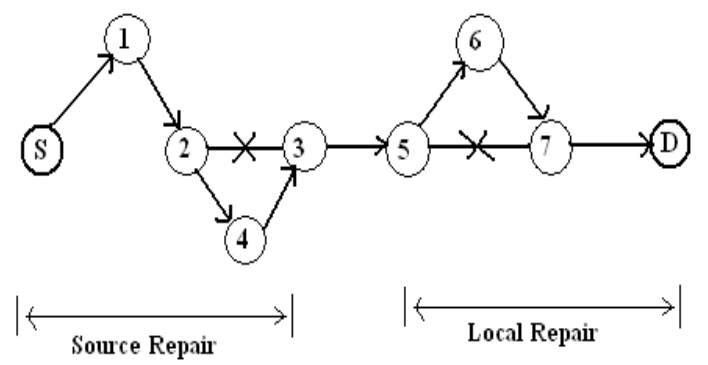

Figure 1 link break during transfer of data

After this using NS-2 simulation performance evaluation of modified AODV with existing AODV shows that AODV is competent to proficiently deal with route failures, arises due to movement of nodes in the network. Specifically, the packet loss has reduced up to $40 \%$ and it achieves a significant improvement in the end-to-end delay. In this proposed approach routing overhead can also be reduced by about 20 to $30 \%$ by minimizing route discovery operations 


\section{RELATED STUDY}

In this paper cluster-Based Multipath Dynamic Source Routing in MANET (CMDSR) is designed which can easily manipulate according to network dynamics [12]. This idea is based on the hierarchy to execute a Route identification and allocation of traffic for multiple diverse paths. In this method basically executed on a multilevel hierarchical method of 1cell cluster and 2-server cluster. In this idea main proposition is to transmit the Route identification method to the 2-server level to avoid the network overflow due to the DSR Route identification.So, Route identification not required overflow or flooding mechanism and minimize the overhead,it improves the networks modification. When overhead increases appreciably with an increasing node compactness and a good number of nodes in the plane composition, i.e modification is hard

This idea proposes an approach based on the ODMRP (OnDemand Multicast Routing Protocol) in MANET,a uniform ODMRP (R-ODMRP) is suggested for mostly used throughput and mainly effective for high-speed MANET, it contains packet acknowledgement, lost data recovery, uniform access and QoS based packet delivery [10]. It includes searching of the active network, R-ODMRP constructs the multicast routing based on the cluster, establishes a distributed mechanism of the acknowledgment and recovery of packet delivery. Along side with the cluster key circulated in one cluster, this method can provide security with consistency of multiple sender and receiver based on local security policy. When we calculate the result of this method is evaluated based on the network simulator and gets a significant enhancement.

In this method, it gives a general analysis of research on local repair of connection, when it is ended during communication for MANET and it proposed a new local repair scheme for making up the lack of connection of the existing local repair schemes. This improves local repair scheme based on the overhead requirement and point to point delay in data execution. Process are mainly contain to keep the next two-hop node address which executes entry in the routing table. During local repair, the repairing node use Ant algorithm for finding new routes for next to next node in the link considering that other part of the link is already in existence. The idea is based on F-ANT and B-ANT will give details of a data packet in overhead [4]. This repairing node not only provide the discovery of a route to the target node of a data packet, it finally attempts to establish the route to its downstream node. The proposed algorithm will be highly adaptable,moderate and efficient, which mainly minimize point to point delay in high mobility cases.

This paper proposed a uniform approach for Neighbor Detection Algorithm for AODV Routing Protocol for the betterment of link feature [7]. This method is used to check the feature of link as a utility of SNR. Link with better quality factor are selected for the communication. In this algorithm mainly effective neighbors are used in routing tables for all routes are established over good quality links. It guarantees that both transmit and receive messages have the same amount of chances of being received correctly regardless based on using transmission rate.

In this approach it suggested a generalized route repair (GRR) technique, which maintenance a route on the fly as soon as it is broken and eliminates the need for network-wide flooding. GRR technique resulted in a huge reduction in overhead when virtual on an event driven packet level simulator and results in nearly 30 percent overhead savings. This performance improves other network characteristics as well.

When we talk about this method as compare with AODV protocol, represents Preemptive Local Route Repair (PLRR) [7], that intend to evade route failures by pre-emptively local repairing routes at a time of link break is occurring. In this protocol, it is enhancing node's information concerning link stability to its neighbors resorting to any messages. In this message it appended with a mobility expansion containing the node's location, action vector and a linked timestamp. This mobility data will be used to guess the instant $a$ link between two neighbors will break. This scheme does not take into description of the sender and receiver location information, as other location aided routing protocols. This proposal aims at improving the AODV Quality of Service capabilities by minimizing route failure.

It proposes a new resolution based method for link repairing \& detection of repairing algorithm. By using AODV in a fixed Local Repair threshold in terms of number of hops from the receiver where a link break occurs, to find invoke Source Repair or Local Repair. It suggested a Local Repair Threshold bound that determines how long the end-to-end path that a link break needs to occur in sequence to initiate Local Repair, as opposed to Source Repair [9]. The reproductive results show that the optimal choice of the Local Repair entry, for Packet Delivery Ratio depends on the network load.

\section{PROBLEM STATEMENT}

In a MANET, it is always self executed, structure-less network of mobile devices which connected without wires. Regular and random network topology changes because of the random movement of the nodes. The AODV (Ad Hoc on Demand Distant Vector Routing) protocol works in a dynamic manner because it establishes a route on demand and continue follow that route until it breaks [11]. The link failure occurs due to the mobility of nodes and heavy traffic of data packets in the network. In existing AODV protocol we can not detect the unpredictable nature of the node. With that unpredictable nature of the node it is not clear which repairing mechanism is to select. At the time of considering the route repairing mechanism a trigger of new route discovery mechanism is given by link failure. That's why we need a preemptive approaches which can detect the link failure prior to its occurrence [15]. Link parameters like packet delivery ratio, node energy, link strength, etc. are doing this work. In case of link failure an active decision, of which algorithm is being selected, will be taken by this proposed work accordingly

\section{$>$ For Loop Free Route Discovery}

$>$ For Reputation Free Routes

$>$ For defensive Route Failure Detection.

$>$ To identify the link Failure stipulation

$>$ For taking the decision of which route repair mechanism

$>$ For an error free routing which saves cost, Time \& Energy

Identified Parameter for Link Decision:- In comparison with the standard AODV, predictive approach will show that a flexible and parameterized approach for selecting Local Repair threshold, packet delivery ratio can be improved around 35 to $40 \%$ (in absolute terms) [13]. In this approach it is clear that using such a predetermined threshold as a basis 
for assessment making is suboptimal, when the most encouraging choice of the threshold depends on the level of network stack. The idea shows clearly that an optimal selection of the threshold can give significant performance improvement, in comparison with preset threshold in AODV, and the only approach taken by DYMO is source repair [15].In this paper, we are going to use a more flexible, parameterized and adaptive route repair strategies. After this it is implemented by MANET and wireless routing protocol such as AODV.

Problem Identification:- This paper, it covered a question, which is one of the important research questions, how to find the optimal Local repair threshold TLR for different kind of networks and different levels of network load. This problem is the main focus of our proposed (TB-PRRA) approach having multi-hop link failure identification for Local repair in AODV.

In an Ad-hoc network it provides uniform devices, limited bandwidth, high resolution of error rates and a regularly changing topology are the main characteristics. Out of these available resources, the most constraining is battery power. Due to this the control overhead, processing overhead, Multihop routing capability and Dynamic topology maintenance should be minimal. Many proactive and reactive routing has been already in existence [17]. In this routing table is organized within the neighborhood to improve response time for local movement and to provide fast response time when a request for the establishment of the new route is arises. The main objectives of this algorithm are [18]:- To distinguish between local connectivity management, neighborhood detection and general topology maintenance. -To broadcast route discovery packets only whenever necessary [14]. When we communicate the information about changes in local connectivity to those neighboring mobile nodes that is likely to need the information.

\section{PROPOSED METHOD}

When we talk about the problem of AODV network our proposed method is best suited in a routing path is constructed when a node needs to communicate with other network in its specific range. It starts with route discovery for data transmission from source to destination. And when the route exists, then information is directly sent otherwise a new route request (RREQ) is being inundated in the network. After that destination node replies with a route reply packet (RREP). After successful retrieval of the route reply new route can be formed.

As we take some cases a few of the link is being disconnected or broken due to environmental factors. Due to data loss that occurs. So, the link needs to be verified before the data transmission. If the link break occurs, then the decision must be taken at the proper time to initiate source repair or local repair mechanism. When we are getting the above mentioned goals this work mainly based on a novel Threshold based preemptive route repair algorithm. The initial source node sends the Hello packet to evaluate the present status on the link \& nodes. In this Hello packet contains Battery Life \& Node mobility field on the basis of which any average threshold value is calculated. After this rank is being given to each link representing their strength. During the data transmission if the value of threshold for this mentioned link is below than the defined average threshold, then the new route discovery is initiated giving the pre-emptive signal of future link break, but the route still continues. Again, when the value of this strength is lower than this minimum (min (threshold)) then the route will be changed and an alarm message is being issued to each node so that updating can be done at the routing table. After this pre-emptive approach decision is taken to initiate the link repair either source or local repair. This decision is based on the network value in distance covered by transmitting the data packet. When the failure occurs at neighbor nodes of source than the source repair is initiated or else the local repair can work smoothly. The proposed algorithm is mentioned below:-

\subsection{Threshold Oriented Preemptive Route Repair Algorithm}

The complete scenario for the proposed approach is executed in 3 step algorithms. These are:-

\section{Shortest Path Algorithm:}

1. Initiate with the Node generation for initial User.

2. Generated nodes are classified by Co-ordinates (A, B).

3. Once this the scenario is generated for communication

4. Each node has a pre-defined range, for which it can find the presence of other nodes. The following strategy is adopted when a node needs to find a path to an unknown destination.

5. Identification of Nodes transmission ranges by 4 Quadrant Clock techniques.

a. Verify the range in Positive Quadrant Clockwise Direction

b. Verify the range in Negative Quadrant Clockwise Direction

c. Verify the range in Down Positive Quadrant Clockwise Direction

d. Verify the range in Down Negative Quadrant Clockwise Direction

6. In this method, it is particularly useful in determining the shortest path (minimum number of hops) to the destination.

\section{Transmission of Data Form Source to Destination} Algorithm:

1. Next, the user requests for the transmission of data from the source to the destination.

2. Node $\mathrm{S}$ wants to communicate with $\mathrm{D}$

3. Initiate route identification mechanism

a. If $\mathrm{D}$ is a neighbor of $\mathrm{S}$

b. Route available for data transmission

4. Otherwise $\mathrm{S}$ Checks its routing table for its neighbor.

5. If no route is present

6. Start new route discovery

7. Source $S$ sends new RREQ to its entire neighbor.

8. This message is flooded till $\mathrm{D}$ receives this.

9. After this D reply with RREP.

10. Communication Starts.

Preemptive Route Repair Algorithm :

1. Each node measures the link strength after every $10 \mathrm{Sec}$.

a. This value gives the threshold on the basis of following parameters

b. Threshold (Battery power, Packet delivery Ratio, TTL Value, No of Packet Relayed, Node Mobility within the range) 
Threshold $=[$ Initial Battery Power $+($ Packet deliver in Unit Time/ Total number of Packet) + Total Time Taken by Packet +Total number of packet relayed+ Mobile Nodes in a network] * (40/100)

2. We then investigate what the optimal choice is of this threshold for a range of network scenarios. We define the link break location parameter $\mathbf{l}_{\mathbf{l b}}$ as follows: $1_{\mathrm{lb}}=$ (hop index of broken link / Total number of hops in Path)

3. Each node shares this information \& gives rank to every other node \& link.

4. If the links condition < min threshold

c. Initiate new route discovery

5. End If threshold < min (min threshold)

d. Issue Route Change Alarm message to source

e. Each node shares this message \& updates their routing table.

\section{PERFORMANCE EVALUATION}

In informative implementation \& result analysis via several graphs \& process monitoring we will show that our proposed protocol(AOMDV) in this paper performs improved than the existing (AODV), in terms of the different performance evaluation measures in the network like packet delivery rate, average hops and packet loss. The proposed modified routing protocol prepares the alternate route whenever there is a link failure occurs between any two nodes in the network

$>$ A pre-emptive link failure will be detect by using above proposed approach

$>$ Link strength can be used as status.

Dy early failure detection loss of data can be saved

$>$ By hello message sensing of channel can be done easily

$>$ Time based transmission can be done

$>$ In routing rank of the link can be used

$>$ Retransmission of data can be minimized

Evaluation of the performance of above proposed approach is shown with the help of following parameters like:

Control overhead: The control overhead is defined as the total number of routing control packets normalized by the total number of received data packets.

Average point to point delay: The point to point dealy is averaged over all surviving data packets from the sources to the destinations.

Packet delivery Ratio: It shows the total number of packet delivered successfully

Battery power: It shows the total battery power consumed while maintaining this pre-emptive link break approach

Node Mobility within the range: It shows the possible occurrence of event related to node motion which also link breaks.

TTL Value: It is the total time required to travel in between intermediate nodes.
No of Packet Relayed: This can be used to analyse the traffic load \& number of packet relayed which causes the link break.

\section{CONCLUSION}

In this schem will shows an enhanced performance \& an improved error free data transmission at different nodes in the network. To achieve better and pre-emptive link repair it is a innovative extension to the modified AODV protocol. In the same way which repairing mechanism is better at what condition can also be decided through the proposed approach (TB-PRRA). This method initiate the alternate route preparation process we do not need to wait for link failure, it could be initiated at any time. The decision about when to direct packets to the alternate route is taken by the upstream nodes with the help of alternate route's information which is provided by the down streams nodes. For that reason it is clear that by applying the threshold based approach when link failure occurs will definitely provide the better result in future. So conclusion is, this type of error free route repair scheme can direct for effective transmission without worrying about the path optimality \& link condition, which has been addressed as a problem in local recovery operations.

\section{FUTURE WORK}

In future proposed scheme can be enhanced in terms of both link repair and other ways of threshold by our proposed approach. This proposed scheme has one limitation it selects the best link repair method on its strength, if any, only between the nodes which are located 2-hop away. So in future our focus would be on developing a link repair mechanism which can detect link repair of any value of nodes in the network.

\section{ACKNOWLEDGMENT}

This research work is self-financed but recommended from the institute so as to enhance the link repair problem with present techniques in Ad Hoc network using this method. Thus, the authors thank the anonymous reviewers for their valuable comments, which strengthened the paper. The authors also wish to acknowledge institute administration for their support $\&$ motivation during this research. They also like to give thanks to Mr. Purushottam kumar for discussion regarding the situational awareness system \& for producing the approach adapted for this paper.

\section{REFERENCE}

[1] Saaidal R. Azzuhri, Marius Portmann \& Wee Lum Tan , "Evaluation of Parameterised Route Repair in AODV" in IEEE Transaction 2011.

[2] Zeki Bilgin \& Bilal Khan "A Dynamic Route Optimization Mechanism for AODV in MANETs " in IEEE Transaction 2010.

[3] S. Revathi \& T. R. Rangaswamy , "Secured Optimal Adaptable Ad-Hoc Routing Protocol in MANET S". in European Journal of Scientific Research Issn 1450-216, Vol.73 No.1, 2012.

[4] Jyoti Jain, Roopam Gupta in T.K. Bandhopadhyay, "On Demand Local Link Repair Algorithm for AODV Protocol “ in IJCA Volume 35- No.5, December 2011.

[5] Rachid Haboub \& Mohammed Ouzzif, "Secure \& reliable routing in MANET" in IJCSES Vol.3, No.1, February 2012. 
[6] Nadia Qasim, Fatin Said \& Hamid Aghvami, "Mobile Ad Hoc Networking Protocols' Evaluation through Simulation for Quality of Service" in IAENG, 36:1, IJCS_36_1_10,February 2009.

[7] P. Priya Naidu1 \& Meenu Chawla, "Extended Ad Hoc on Demand Distance Vector Local Repair Trial for MANET" in IJWMN, Vol. 4, No. 2, April 2012.

[8] K.Muthumayil, V.Rajamani \& S.Manikandan, “ Ad-Hoc Cross Layered Energy based on-demand Routing Protocol for MANETs" in Conference in R.M.D Engg.College, Gummudipoondi, Dec-2011.

[9] Fikret Sivrikaya, Sahin Albayrak \& Bulent Yener, "Spatially Limited Contention for Multi-Hop Wireless Networks" in IEEE Globecom Issn 978-1-4244-23248/08 IEEE. 2008

[10] Zhiming $\mathrm{Xu}, \mathrm{Yu}$ Wang \& Jingguo Zhu, “A Reliable Multicast Routing Protocol for High-speed Mobile Ad Hoc Networks: R-ODMRP" in Journal of Software, Vol 9, No 2, 2009.

[11] S. Subburam and P. Sheik Abdul Khader, "Efficient Two Hop Local Route Repair Mechanism Using QosAware Routing for Mobile Ad Hoc Networks" in Indian Journal of Science and Technology ISSN:0974-6846 Vol:5 Issue:11 November 2012
[12] Hui-Yao An, Ling Zhong, Xi-Cheng Lu, and Wei Peng, "A Cluster-Based Multipath Dynamic Source Routing in MANET" in IEEE Transaction, 2005.

[13] S. Revathi \& T. R. Rangaswamy , "Dynamic Route Shortening and Route Repairing Mechanism for Mobile Ad Hoc Networks" in Journal of Computer Science ISSN 1549-3636 8 (8): 1212-1218, 2012.

[14] Jianlin Guo, Chuan Han, Philip Orlik \& Jinyun Zhang, "Loop-Free Routing in Low-Power and Lossy Networks" in Sensorcomm IARIA, ISBN: 978-1-61208207-3, 2012.

[15] J.-N. LIU and Imrich Chlamtac "Mobile ad hoc networking with a view of $4 \mathrm{G}$ wireless: Imperatives and Challenges".

[16] C. S. R. Murthy and B. Manoj "Ad Hoc Wireless Networks: Architectures and Protocols", Prentice Hall PTR, May 2004.

[17] Elizabeth.M.Royer, C-K Toh,” A Review of Current Routing Protocols for Ad-hoc Mobile Wireless networks",IEEE personal communication,pp.46-55,April 1999.

[18] C. E. Perkins, E. M. Royer, and S. R. Das, "Ad hoc ondemand distance vector routing," IETF RFC 3561, July 2003 